\title{
PDE-SVD BASED AUDIO DENOISING
}

\author{
George Baravdish, Gianpaolo Evangelista, Olof Svensson \\ Faten Sofya* \\ Linköping University \\ Norrköping, Sweden
Mosul University
Mosul, Iraq

\begin{abstract}
In this paper we present a new method for denoising audio signals. The method is based on the Singular Value Decomposition (SVD) of the frame matrix representing the signal in the Overlap Add decomposition. Denoising is performed by modifying both the singular values, using a tapering model, and the singular vectors of the representation, using a nonlinear PDE method. The performance of the method is evaluated and compared with denoising obtained by filtering.
\end{abstract}

Index Terms - Denoising, Audio Restoration, Speech Enhancement, Singular Value Decomposition, Partial Differential Equations

\section{INTRODUCTION}

Denoising is an important component of audio restoration systems that aims at recovering sound documents recorded in the past. Noise is introduced by the original analog recording systems, by transfer to other storage media and by the ageing of the media. Denoising is relevant for speech communication in noisy environments to improve intelligibility and enhance quality.

Several approaches have been proposed for audio and speech denoising in time, frequency and time-frequency domains [1, 2, 3, 4]. In this paper, we present an approach based on a time domain (TD) matrix representation of the signal. By means of a sliding window, the noisy signal is decomposed into fixed length partially overlapping frames that are collected in a matrix. The signal matrix is exactly represented by means of the Singular Value Decomposition (SVD) [5]. An approach to directly denoising the signal by retaining the principal components (lower rank from highest singular values) in the SVD proved unfruitful since the signal appears highly degraded even though some nosie reduction occurs.

In the effort to reduce the contribution of the noise energy without destroying the signal, the singular values are tapered according to a threshold and a model for the energy decay of the high order singular values, which are less relevant for the signal.

\footnotetext{
*Thanks to the Scholar Rescue Fund agency for funding and Linköping University for generous hospitality
}

The singular vectors, which contain traces of the time domain contribution of the noise, are denoised by means of a Partial Differential Equation (PDE) method, which is inspired by a denoising technique previously applied by the authors to image signals [6]. The PDE stems from a minimization problem attempting to obtain singular vectors closest to the unknown signal singular vectors, with a penalty term ensuring smooth gradient. Both linear and nonlinear solution methods are considered, with the nonlinear case providing best results at an increased computational cost.

The results show good performance of the algorithm in terms of objective and subjective measures. In our preliminary tests, an increase of SNR is achieved when the signal is highly corrupted, i.e. when the original SNR is in the $0 \mathrm{~dB}$ or negative range, which makes the method suitable for denoising highly degraded signals.

The paper is organized as follows. In Section 2 we review PDE based methods for denoising, in Section 3 we describe the SVD denoising approach, in Section 4 we show the results of some of our tests. Finally, in Section 5 we draw our conclusion and discuss further improvements.

\section{DENOISING WITH PDE}

It is by now well known that Partial Differential Equations (PDE) can be used to denoise signals. For methods for denoising sounds using PDE see, e.g., [7], [8], [2] and [9]. For techniques for denoising images see [10], [11], [12] and [13]. Recently, Baravdish and Svensson [6], have proposed a method to denoise images based on nonlinear PDE.

In this paper we are going to use methods similar to those in [6] to denoise two dimensional data derived from sound, as briefly described in Section 2.

The PDEs considered in our method are special cases of evolution equations. For a background on this topic see [14].

\subsection{PDE Methods}

Assume that $u$ is a bidimensional clean original data and $u_{0}$ is a noisy version of $u$ such that $u_{0}=u+n$ where $n$ is the noise. To denoise the data $u_{0}$ one minimizes the functional

$$
J(u)=\int_{\Omega}\left(\frac{1}{2}\left(u-u_{0}\right)^{2}+\lambda \Phi(|\nabla u|)\right) d \mathbf{x}, \lambda>0
$$


where $\Phi$ is a strictly convex function. In the energy functional $J$, the first term measures the fidelity to the noisy data and the second term imposes a smoothness condition on the clean data $u$. The Euler-Lagrange equation associated with the energy functional $J$ is the partial differential equation (PDE) $\frac{\partial J}{\partial u}$ with Neumann boundary condition zero on the boundary of $\Omega$.

By introducing a "time" variable, we instead solve a time dependent PDE $u_{t}^{\prime}=-\frac{\partial J}{\partial u}$ with the noisy data $u_{0}$ as initial data and with zero Neumann boundary condition. Assume that $\Phi(|\nabla u|)=\frac{1}{p}|\nabla u|^{p}, 1 \leq p<\infty$. In the case that the exponent $p=2$ we get the heat equation and for $p=1$ we get the total variation approach of Rudin, Osher and Fatemi [13]. Running the time dependent PDEs forward in time gives a cleaner data than the initial noisy version $u_{0}$. Thus, we need a stopping time $T$ to stop the process.

\subsection{Inverse PDE}

In the method in [6], it is assumed that the given degraded data $u_{0}$ is given at a later time $T$ and the original data $u$ is sought for at time $t=0$. This is an inverse problem, which is backward in time. The solution is obtained by considering a sequence of well-posed problems. Each problem is a forward version where the initial data is chosen properly to reconstruct the original data $\mathrm{u}$. The aim of this approach is twofold. By solving the PDE forward in time smoother data is obtained which is cleaner and the choice of the initial data is such that the data also get de-blurred.

Hence, instead of solving the forward problem, the following inverse problem is solved,

$$
\left\{\begin{array}{llll}
\partial_{t} u-\operatorname{div}\left(|\nabla u|^{p-2} \nabla u\right) & = & 0 & \\
\partial_{n} u(\mathbf{x}, t) & = & 0 & \partial \Omega \times(0, T) \\
u(\mathbf{x}, 0) & = & \varphi(\mathbf{x}) & \mathbf{x} \in \Omega
\end{array}\right.
$$

where $u$ and $\varphi$ are unknown and an additional data

$$
u(\mathbf{x}, T)=\psi
$$

is given. The idea here is that the noisy data $u(\mathbf{x}, T)=\psi$ is given at a later time and we want to reconstruct the original data at an earlier time: $u(\mathbf{x}, 0)=\varphi$. There exists a nonlinear operator $A$ such that the inverse problem in Eq. (1) and (2) can be reduced to a nonlinear operator equation

$$
A(\varphi)=\psi
$$

There are by now several methods of solving the operator equation Eq. (3). One of them is the nonlinear Landweber method which starts with an arbitrary $\varphi_{0}$ :

$$
\varphi_{k+1}=\varphi_{k}-A^{*}\left(A \varphi_{k}-\psi\right)
$$

where $A^{*}$ is the adjoint Frechet derivative of $A$. For the convergence rates and results on this method, see Engl, Hanke and Neubauer [15]. To solve the problem in (1) and (2), the following iterative method was proposed in [6]. Let $\varphi_{0} \in L^{2}(\Omega)$ be arbitrary. Assume that $u_{k}$ has been constructed. Then we proceed to solve the linear adjoint problem

$$
\begin{cases}\partial_{t} v_{k}+\operatorname{div}\left(L\left(\left|\nabla u_{k}\right|\right) \nabla v_{k}\right) & =0, \quad \Omega \times(0, T) \\ \partial_{n} v_{k}(\mathbf{x}, t) & =0, \quad \partial \Omega \times(0, T) \\ v_{k}(\mathbf{x}, T) & =u_{k}(\mathbf{x}, T)-\psi(\mathbf{x}), \mathbf{x} \in \Omega\end{cases}
$$

where

$$
L_{\varepsilon}(|\nabla u|)=|\nabla u|^{p-2} I+(p-2)|\nabla u|^{p-4} \nabla u \nabla u^{t} .
$$

For $k+1$ solve

$$
\begin{cases}\partial_{t} u_{k+1}-\operatorname{div}\left(|\nabla u|^{p-2} \nabla u\right) & =0, \quad \Omega \times(0, T) \\ \partial_{n} u_{k+1}(\mathbf{x}, t) & =0, \quad \partial \Omega \times(0, T) \\ u_{k+1}(\mathbf{x}, 0) & =u_{k}(\mathbf{x}, 0)-v_{k}(\mathbf{x}, 0), \mathbf{x} \in \Omega\end{cases}
$$

Notice that for obvious numerical reasons, the $|\nabla u|$ is considered as a regularized version $\sqrt{|\nabla u|^{2}+\delta^{2}}$, where $\delta$ is some fixed small number. This scheme is in analogue with the one given in Eq. (4).

\section{OPTIMIZED PDE-SVD DENOISING}

The proposed technique aims to reduce additive random noise present in the signal. It is based on the singular value decomposition (SVD) of the matrix representing the noisy signal in the time domain (TD). In our approach the noisy signal is transformed into a matrix of partially overlapping regularly spaced windowed signal segments.

By thresholding, the singular value matrix can be partitioned into two blocks, pertaining to two subspaces, the signal subpace and the noise subspace. Clearly, the connotation of signal and noise subspace is not strict, the signal subspace is the one that mostly contains the signal and the noisy subspace is the one that mostly contains noise. Since the singular values are intrinsic properties of the matrices, part of the denoising strategy is to reobtain the singular values of the original "clean" signal. The aim is to subtract from the singular values of the noisy signal the estimated singular values of the noise. We perform this operation on higher index singular values where the contribution of the signal is smaller.

The singular values pertaining to the noise subspace are tapered using an algorithm given in this section. Denoising is refined by smoothing the matrices of the singular vectors in the SVD representation using the inverse PDE method in [6]. The denoised signal is reconstructed using the smoothed singular vectors and the altered singular values.

Here below we describe the method in further detail.

\subsection{Framing the noisy signal into a matrix}

We divide the signal into $M$ frames each of length $N$ with half-length overlap. To each of these frames we apply a time 
window, e.g. the von Hann or the rectangular window. The $M \times N$ matrix $A_{n}$ is the collection of the windowed signal frames each arranged in a different row.

\subsection{Singular value decomposition (SVD)}

The SVD of the $M \times N$ matrix $A_{n}$ is given by

$$
A_{n}=U \Sigma V^{T}
$$

where $U$, an $M \times M$ matrix, and $V$, an $N \times N$ matrix, are orthogonal matrices, and $\Sigma$ is an $M \times N$ diagonal matrix of singular values (SVs) $\left(\Sigma_{i j}=0\right.$, if $i \neq j$ and $\Sigma_{11} \geq \Sigma_{22} \geq$ $\cdots \geq 0$ ). We will denote $\Sigma_{i i}$ by $\sigma_{i}$.

The columns of the orthogonal matrices $U$ and $V$ are called the left and right singular vectors, respectively. The singular values $\left\{\sigma_{i}\right\}$ represent the importance of individual singular vectors in the composition of the matrix. In other words, larger singular values have more information about the structure of patterns embedded in the matrix than the smaller singular values.

\subsection{Splitting $\Sigma$ by thresholding}

Using a threshold index $\tau$ the singular value matrix can be partitioned into two blocks, $\Sigma_{s}$ and $\Sigma_{n}$ as follows:

$$
\Sigma=\left(\begin{array}{cc}
\Sigma_{s} & 0 \\
0 & \Sigma_{n}
\end{array}\right) .
$$

The threshold index $\tau$ can be computed as the center of mass for the singular values, defined by

$$
\tau=\frac{\sum k \sigma_{k}}{\sum \sigma_{k}} .
$$

The block $\Sigma_{n}$ contains the singular values $\sigma_{k}$ such that $k \geq$ $M^{\prime}=\lfloor\tau\rfloor$.

In Figure 1(a) a plot of the clean and noisy singular values is shown, together with the result of thresholded constant tapering. There, the solid line represents the singular values of the clean signal, the broken line the singular values of the noisy signal and the dotted line the tapered singular values used for denoising. The vertical segment shows the position of the center of mass threshold.

The center of mass choice for the tapering threhold proved to be too conservative in our tests. A certain quantity of noisy singular values still persists after tapering. For this reason we resorted to a threshold value computed as a fraction of the center of mass. In Figure 1(b), the effect of selecting the tapering threshold as $10 \%$ of the center of mass is shown, with the same line notation as in Figure 1(a). It is clear that the tapered values in this case better follow the clean singular values curve.

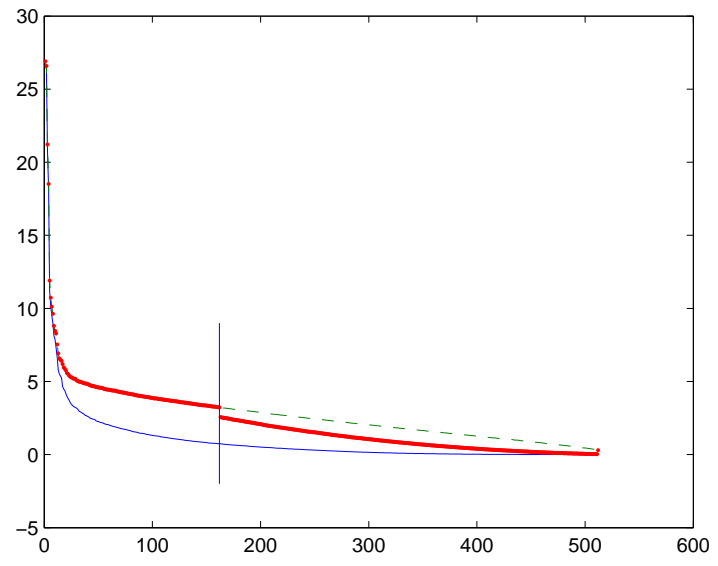

(a)

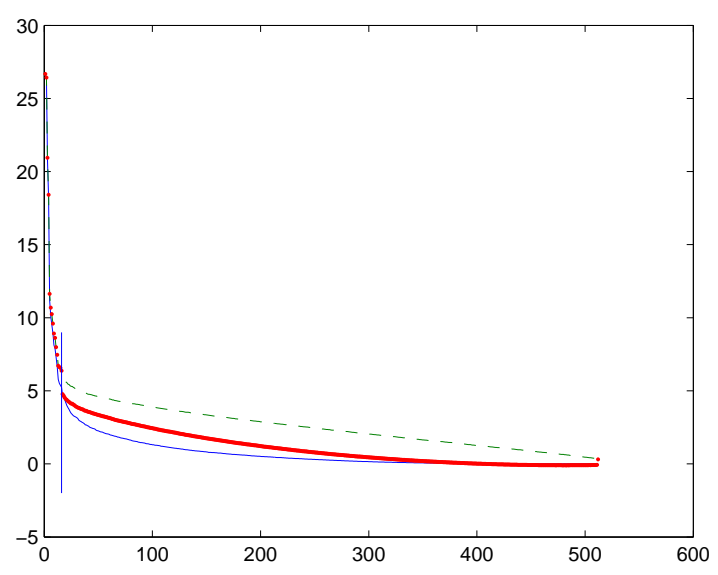

(b)

Fig. 1. Examples of thresholding and constant tapering of the singular values: (a) Center of mass choice for the threshold; (b) $10 \%$ of the center of mass choice for the threshold.

\subsection{Modifying $\Sigma_{n}$}

In this section we will try to reduce the contribution of noise to the singular values in $\Sigma_{n}$ in (5). We denote by $\sigma_{n, 1} \geq$ $\sigma_{n, 2} \geq \cdots \geq 0$ the singular values in $\Sigma_{n}$.

Here we are interested in modifying the singular values in the following manner: $\sigma_{k}^{*}=\alpha \sigma_{n, k}$, where $\alpha$ is any positive function of $k$.

If we replace the noisy singular values in $\Sigma_{n}$ by the denoised values $\sigma_{k}^{*}$, we obtain another diagonal matrix $\Sigma_{n}^{*}$. Substituting for this matrix in (5) yields the following estimate for the singular value matrix of the clean signal $\Sigma_{c}$ :

$$
\Sigma_{d}=\left(\begin{array}{cc}
\Sigma_{s} & 0 \\
0 & \Sigma_{n}^{*}
\end{array}\right) .
$$


The MSE measure for the SVs gives

$$
\begin{aligned}
\left\|\Sigma_{c}-\Sigma_{d}\right\|^{2} & =\sum_{1}^{M^{\prime}-1}\left|\sigma_{k}^{c}-\sigma_{k}^{*}\right|^{2}+\sum_{M^{\prime}}^{M}\left|\sigma_{k}^{c}-\sigma_{k}^{*}\right|^{2} \\
& =\sum_{1}^{M^{\prime}-1}\left|\sigma_{k}^{c}-\sigma_{k}^{*}\right|^{2}+\sum_{M^{\prime}}^{M}\left|\sigma_{k}^{c}-\alpha \sigma_{n, k}\right|^{2} .
\end{aligned}
$$

In the case of constant $\alpha$, the right hand side in (8) is minimized by chosing

$$
\alpha=\frac{\sum_{k=M^{\prime}}^{M} \sigma_{k}^{c} \cdot \sigma_{n, k}}{\sum_{k=M^{\prime}}^{M}\left(\sigma_{n, k}\right)^{2}} .
$$

The value of $\alpha$ in (9) yields a lower upper bound on the MSE in our proposed method for the case when $\sigma_{k}^{c}$ is known.

In the case of unknown clean signal we proceed from (9) as follows. By using the Cauchy-Schwarz inequality we obtain the estimate

$$
\alpha=\frac{\sum_{k=M^{\prime}}^{M} \sigma_{k}^{c} \cdot \sigma_{n, k}}{\sum_{k=M^{\prime}}^{M}\left(\sigma_{n, k}\right)^{2}} \leq \frac{\left(\sum_{k=M^{\prime}}^{M}\left(\sigma_{k}^{c}\right)^{2}\right)^{1 / 2}\left(\sum_{k=M^{\prime}}^{M}\left(\sigma_{n, k}\right)^{2}\right)^{1 / 2}}{\sum_{k=M^{\prime}}^{M}\left(\sigma_{n, k}\right)^{2}}
$$

Since the additive noise increases the energy then $\sum_{k=M^{\prime}}^{M}\left(\sigma_{k}^{c}\right)^{2}<\sum_{k=M^{\prime}}^{M}\left(\sigma_{n, k}\right)^{2}$. The choice of $\alpha$ is therefore limited to the interval $0<\alpha<1$.

\subsection{Denoising the Orthogonal Matrices}

We use the nonlinear inverse PDE method to reduce the noise present in the two orthogonal matrices $U$ and $V$ in (2.2), thus obtaining an estimate for the clean matrices $U_{d}$ and $V_{d}$. These matrices are normalized in order to limit energy change due to the PDE denoising procedure. After denoising $\Sigma, U$ and $V$, we get the enhanced TD matrix as follows:

$$
\hat{A}=U_{d} \Sigma_{d} V_{d}^{T}
$$

\subsection{Summary of the method}

The proposed noise reduction technique can be summarized as follows:

1. Use windowing in TD to transform the noisy signal $s_{n}$ into a matrix $A_{n}$ whose rows host the partially overlapping signal segments.

2. Represent the matrix $A_{n}$ with the SVD, obtaining $A_{n}=U \Sigma V^{T}$.

3. Split $\Sigma$ into signal and noise blocks according to the threshold derived in Section 3.3. Modify the noise block according to the method in Section 3.4.
4. Denoise $U$ and $V$ by the PDE method in the Section 2 and normalize matrices.

5. Compose the modified SVs and normalized denoised matrices to obtain the enhanced TD matrix $\hat{A}$ as in (10).

6. The noise reduced signal $s_{d}$ is obtained from the matrix $\hat{A}$ by Overlap Add.

\section{PERFORMANCE EVALUATION}

In order to test our method we performed tests on a variety of sounds from speech and music, corrupting them with additive gaussian noise. We use the sampling rate $16 \mathrm{kHz}$ for speech and $44.1 \mathrm{kHz}$ for music.

The time domain signal frame matrix is obtained by sliding a length 512 samples rectangular window over the signal, using half length overlap. After denoising, the signal is reconstructed by overlap adding the PDE-SVD denoised frames using the von Hann window.

For the parameters in the PDE we used times $T$ in the order of $10^{-2}$ and a value $p=1.6$ for the exponent (see Section 2 ). These values were selected after some testing on performance of the PDE denoising procedure.

As quality measures, we use the Mean Square Error (MSE), defined as

$$
M S E=\frac{1}{N} \sum_{i=1}^{N}\left(s_{c}(i)-s_{d}(i)\right)^{2}
$$

and the Signal to Noise Ratio (SNR)

$$
S N R=20 \log _{10}\left(\frac{\left\|s_{d}\right\|}{\left\|s_{d}-s_{c}\right\|}\right)
$$

where $s_{c}$ is the original signal and $s_{d}$ is the denoised signal, with $\|s\|^{2}=\sum_{i} s(i)^{2}$. We also considered the PEAQ measure [16], however it did not seem to provide sufficient information.

In Table 1 an excerpt of out tests is shown, where three signals are considered: two speech signals "thanks" and "welcome" and a rock music sample "music". We compare our PDE-SVD method with the Savitzky-Golay (SG) filtering [17] (last column to the right) for several values of the SNR ranging from $10 \mathrm{~dB}$ down to $-5 \mathrm{~dB}$ (highly corruptes signal), as reported in the first column from the left. For the PDESVD method we consider different variants for the singular values matrix: center of mass thresholding, with constant tapering $\alpha$, shown in the second column from the left (CM), a less conservative thresholding calculated as $10 \%$ of the center of mass shown in the third column $(10 \% \mathrm{CM})$ and, as a reference, the case where in the PDE-SVD method we assume the clean singular values to be known, i.e. where only the singular vectors are denoised, shown in fourth column from the left. The results concerning the MSE are not reported in the 
table since they lead to similar conclusions as those for the SNR.

SG filtering performed better than the empirical Wiener filter in both objective and subjective tests; for this reason it was retained as a benchmark in the table. The results show that the PDE-SVD method outperforms the SG filtering in most cases, with near equivalence in the very low SNR case. Additionally, as the known singular values case shows, the PDE-SVD method is still subject to improvement deriving from the application of a more refined strategy in the estimation of the clean singular values from the noisy ones. Moreover, extensive optimization of the PDE denoising parameters and model is bound to improve performance, which will be the object of further work.

In our listening tests we concluded that the background noise was clearly reduced and the speech signal is more intellegible. The bandwidth of the denoised music signals is wide and details are better preserved compared to SG filtering.

\begin{tabular}{|c|c|c|c|c|c|}
\hline Sound & SNR & CM & $10 \%$ CM & Known SV & SG \\
\hline speech & 10 & 12.2 & 12.4 & 13.5 & 11.9 \\
"thanks" & 5 & 7.5 & 9.5 & 9.6 & 8.1 \\
& 0 & 3.7 & 6.1 & 7.2 & 4.5 \\
& -5 & 1.4 & 2.4 & 4.1 & 2.6 \\
\hline speech & 10 & 11.7 & 11.9 & 12.7 & 9.9 \\
"welcome" & 5 & 7.0 & 8.2 & 9.0 & 7.2 \\
& 0 & 3.4 & 4.4 & 5.8 & 4.2 \\
& -5 & 1.3 & 1.7 & 3.1 & 1.9 \\
\hline music & 10 & 11.9 & 12.7 & 12.8 & 12.9 \\
"rock" & 5 & 7.2 & 9.0 & 9.3 & 8.6 \\
& 0 & 3.5 & 5.1 & 6.3 & 4.8 \\
& -5 & 1.3 & 2.0 & 3.9 & 2.2 \\
\hline
\end{tabular}

Table 1. Results of the denoising tests on signals speech and music at different SNR (in $\mathrm{dB}$ ).

\section{CONCLUSIONS AND DISCUSSIONS}

In this paper we proposed a new approach for reducing the noise from signal in which the signal is represented by the SVD of the frame matrix. We enhanced the singular values by tapering and the singular vectors of the noisy signal by means of a nonlinear inverse PDE method. We compared our method with Savitzky-Golay filter in terms of MSE and SNR. The results show that our method has good performance in signal noise reduction.

Our results can be further improved by considering a tighter approach to the estimation of the singular values of the clean signal from the singular values of the noisy signal. A probabilistic approach is currently under study. Further improvements will also derive from the adaptation and optimization of the PDE model using a database of sound for benchmarking.

\section{REFERENCES}

[1] Simon J. Godsill and Peter J.W. Rayner, Digital Audio Restoration, Springer, 1998.

[2] Arthur Szlam, "Non-local means for audio denoising," Tech. Rep. 56, UCLA CAM Report 08-56, University of California, Los Angeles, CA, 2008., 2008.

[3] Guoshen Yu, Stéphane Mallat, and Emmanuel Bacry, "Audio denoising by time-frequency block thresholding," IEEE Trans. on Signal Processing, vol. 56, no. 5, pp. 1830-1839, May 2008.

[4] Y. Ephraim, H. Lev-Ari, and W. J. J. Roberts, "A brief survey of speech enhancement," in The Electronic Handbook. CRC Press, Boca Raton, FL, 2005.

[5] Amin Zehtabian and Hamid Hassanpour, "Optimized singular vector denoising approach for speech enhancement," Iranica Journal of Energy \& Environment, vol. 2, no. 2, pp. 166-180, 2011.

[6] G. Baravdish and O. Svensson, "Image reconstruction with $p(x)$-parabolic equations," in Proceedings of ICIPE 2011, The $7^{\text {th }}$ international conference on inverse problems in engineering, A. Kassab and E. Divo, Eds. 2011, Centercorp Publishing.

[7] Benjamín Dugnol, Carlos Fernández, Gonzalo Galiano, and Julián Velasco, "On pde-based spectrogram image restoration. application to wolf chorus noise reduction and comparison with other algorithms," in Signal Processing for Image Enhancement and Multimedia Processing, vol. 31 of Multimedia Systems and Applications Series, pp. 3-12. Springer, 2008.

[8] Yingyong Qi and Jack Xin, "A perception- and PDEbased nonlinear transformation for processing spoken words," Phys. D, vol. 149, no. 3, pp. 143-160, 2001.

[9] Mohsen Nikpour and Hossein Ashtiani, "Using pde's for noise reduction in time series," International Journal of Computing and ICT Research, vol. 3, no. 1, pp. 20422048, 2009.

[10] Luis Alvarez, Pierre-Louis Lions, and Jean-Michel Morel, "Image selective smoothing and edge detection by nonlinear diffusion. II," SIAM J. Numer. Anal., vol. 29, no. 3, pp. 845-866, 1992.

[11] Antonin Chambolle and Pierre-Louis Lions, "Image recovery via total variation minimization and related problems," Numer. Math., vol. 76, no. 2, pp. 167-188, 1997.

[12] Pierre Kornprobst, Rachid Deriche, and Gilles Aubert, "Image sequence analysis via partial differential equations," J. Math. Imaging Vision, vol. 11, no. 1, pp. 5-26, 1999. 
[13] L. Rudin, S. Osher, and E. Fatemi, "Nonlinear total variation based noise removal algorithms," Physica D, vol. 60, pp. 259-268, 1992.

[14] E. Di Benedetto, Degenerate parabolic equations, Springer Verlag, New York, 1993.

[15] Heinz Engl, Werner Hanke, and Martin A. Neubauer, Regularization of inverse problems, Springer Verlag, 1996.

[16] Thilo Thiede, William C. Treurniet, Roland Bitto, Christian Schmidmer, Thomas Sporer, John G. Beerends, and Catherine Colomes, "Peaq - the itu standard for objective measurement of perceived audio quality," J. Audio Eng. Soc, vol. 48, no. 1/2, pp. 3-29, 2000.

[17] A. Savitzky and M.J.E. Golay, "Smoothing and differentiation of data by simplified least squares procedures," Analytical Chemistry, vol. 36, no. 8, pp. 1627-1639, 1964. 